\title{
Serum alkaline phosphatase isoenzymes in laboratory beagle dogs detected by polyacrylamide-gel disk electrophoresis
}

\author{
Kazuhisa Hatayama, Yoshito Nishihara, Sayaka Kimura, Ken Goto, Daichi Nakamura, \\ Atsushi Wakita and Yoshinaka Urasoko \\ Toxicology Department, Gotemba Laboratory, Bozo Research Center Inc., 1284 Kamado, Gotemba-shi, \\ Shizuoka 412-0039, Japan
}

(Received April 18, 2011; Accepted June 27, 2011)

\begin{abstract}
Serum alkaline phosphatase (ALP) activity is frequently measured in toxicity studies. Itoh et al. (2002) reported that a commercially available polyacrylamide-gel (PAG) disk electrophoresis kit used in humans (AlkPhor System, Jokoh Co., Ltd., Tokyo, Japan) for identifying serum ALP isoenzymes was useful for veterinary clinicopathological diagnosis in mongrel dogs. In the present study, based on the report of Itoh et al. (2002), we tried to expand the application range of this kit to laboratory beagle dogs which are commonly used in toxicity studies. In order to identify the origin of each ALP isoenzyme, tissue ALP extracts from the liver, bone and small intestine and serum samples were treated with neuraminidase, anti-small intestinal ALP antibody, ALP inhibitor levamisole and/or wheat germ agglutinin (WGA). The main serum ALP isoenzymes in 5-month-old intact beagle dogs were bone-derived (bone and atypical ALP: corresponding to human variant bone ALP) and they tended to decrease with age. However, liver-derived ALP isoenzyme greatly increased in the serum of cholestasis model dogs. The cholestasis model dogs also had a large molecular ALP detected in the resolving gel. This ALP could be originated from intestinal ALP or corticosteroid-induced ALP (CALP), because the activity remained even after levamisole inhibition. CALP was observed in intact laboratory beagle dogs with individual differences. These results suggest that the present method is a useful tool for detecting serum ALP isoenzymes in laboratory beagle dogs and concomitant levamisole inhibition with another gel is applicable for the evaluation of organ toxicity.
\end{abstract}

Key words: Alkaline phosphatase, ALP, Isoenzyme, Beagle dog, PAG

\section{INTRODUCTION}

Serum alkaline phosphatase (ALP) (orthophosphoric monoester phosphohydrolase; E.C. 3.1.3.1) activity is one of the clinical parameters frequently measured in toxicity studies. Human serum ALP is generally composed of liver-, bone- and intestine-derived isoenzymes, and also a placenta-derived one in pregnant women (Moss, 1992). Recently we reported that the main plasma ALP isoenzyme in intact ICR mice which are frequently used in toxicity studies was bone-derived (Hatayama et al., 2011). Identification of each ALP isoenzyme in serum is important to elucidate the damaged tissue. In the field of veterinary medical science, a lot of examinations have been done on serum ALP isoenzymes, mainly for the diagno- sis of pet dog diseases and their therapy. Syakalima et al. (1998) and Itoh et al. (2002) reported that liver, bone and corticosteroid-induced ALP (CALP) exist in dog serum, while ALP isoenzymes normally found in tissues such as kidney, placenta and intestine have never been demonstrated in dog serum.

Electrophoresis is one of the methods for detecting ALP isoenzymes. Polyacrylamide-gel (PAG) disk electrophoresis has a particularly excellent separating ability (Dingjan et al., 1973). However, it requires a time-consuming procedure and a skillful technique for good reproducibility, and therefore, it had not extensively been used to analyze ALP isoenzymes in animal studies (Syakalima et al., 1998). In the examination using mongrel dogs for the analysis of dog serum ALP isoenzymes, Itoh et al.

Correspondence: Kazuhisa Hatayama (E-mail: hatayama@bozo.co.jp) 
(2002) reported that a commercially available PAG disk electrophoresis kit (AlkPhor System, Jokoh Co., Ltd., Tokyo, Japan) for human ALP analysis was useful for veterinary clinicopathological diagnosis.

In the present study, based on the report of Itoh et al. (2002), we tried to expand the application range of this kit to the evaluation of serum ALP isoenzymes in laboratory beagle dogs which are commonly used in toxicity studies. In addition, in order to ensure steady identification of each isoenzyme, characteristics of the tissue extract ALP and serum ALP were examined in more detail by the method more specific to laboratory beagle dogs in the present study.

All procedures of this study were conducted in compliance with the ongoing official guidelines of animal welfare and according to the protocol approved by the Animal Care and Use Committee at BOZO Research Center Inc. All efforts were made to minimize animal suffering.

\section{MATERIALS AND METHODS}

\section{Animals}

Male and female laboratory beagle dogs were obtained from Covance Research Products, Inc., Cumberland, VA, USA (HRA beagle) at 5 months old and maintained in our laboratory. The animals were housed individually in metal cages in air-conditioned animal rooms (temperature: 18 to $26^{\circ} \mathrm{C}$; relative humidity: 30 to $80 \%$; lighting: $12 \mathrm{hr}$ illumination per day) and provided a pelleted diet (DS-A, Oriental Yeast Co., Ltd., Tokyo, Japan) and tap water ad libitum. For cholestasis model dogs, alpha-naphthylisothiocyanate (ANIT) (Kanto Chemical Co., Inc., Tokyo, Japan) was administered orally to 7 male dogs at 10 to 12 months old as we previously reported (Hoshiya et al., 2002). For the preparation of tissue ALP extracts, 2 male and female intact dogs at 10 months old were used for bone and intestinal samples, and the above-mentioned 2 cholestasis model dogs which would show high liver ALP activity were used for liver samples. Liver, bone and small intestine (duodenum and jejunum) were excised and frozen at $-80^{\circ} \mathrm{C}$ until use. Tissue ALP extracts were prepared as described previously (Hatayama et al., 2011). Blood samples were collected from a total of 63 male and 48 female dogs at 5 to 56 months old ( $n=1$ to 12 for each month of age for each sex) with syringes via the cephalic vein and centrifuged (approximately $1,600 \times \mathrm{g}$ for $10 \mathrm{~min}$ ) to obtain serum. Serum samples were frozen at $-80^{\circ} \mathrm{C}$ until use.

\section{Experimental designs}

In order to obtain the marker for the identification of serum ALP isoenzymes, tissue ALP extracts from the liv- er, bone and small intestine were analyzed by basal electrophoresis, neuraminidase electrophoresis, anti-intestinal ALP antibody electrophoresis, and levamisole electrophoresis. In order to identify serum liver, bone, intestinal and corticosteroid-induced ALP isoenzymes, serum samples obtained from 2 male and 2 female intact dogs at 5 and 11 months old, and 7 male cholestasis model dogs were analyzed by basal electrophoresis, wheat germ agglutinin (WGA) electrophoresis, neuraminidase electrophoresis, levamisole electrophoresis, anti-intestinal ALP antibody electrophoresis, and/or the combination of these electrophoresis.

In addition, the characteristics of serum ALP isoenzymes of laboratory beagle dogs was examined by the methods described below. Although human bone ALP has been reported to be precipitated with WGA (Rosalki and Foo, 1984; Behr and Barnet, 1986), it was required to confirm this reaction in dog serum. In order to examine the existence of WGA-reactive ALP isoenzymes in dog serum, the remaining rate of ALP activity was measured in the supernatant after treatment of serum obtained from 2 male and 2 female intact dogs at 6 months old with $1-10 \mathrm{mg} / \mathrm{ml}$ WGA. Since an increase in small intestinal ALP is associated with ingestion of high fat diets in humans (Matsushita et al., 2002), it was necessary to confirm the effects of feed on dog serum ALP activity. In order to examine the influence of food intake on serum ALP isoenzymes in dogs, ALP activity was examined in serum samples obtained from 6 male and 10 female dogs before and after $17 \mathrm{hr}$ fasting. In the examination of serum liver, bone and intestinal ALP isoenzymes, CALP was detected only in a small number of animals. In order to examine the individual differences in the appearance of CALP, the correlation between the serum ALP activity and that remaining after levamisole inhibition was examined on serum samples obtained from 46 male and 32 female intact dogs at 5 to 56 months old. Samples which markedly deviated upwards from the regression line ( 5 males and 7 females) were examined by basal electrophoresis and levamisole electrophoresis.

\section{Electrophoresis}

Electrophoresis was performed with a commercially available ALP isoenzyme analysis kit, AlkPhor System for humans (Jokoh Co., Ltd., Tokyo, Japan) for PAG disk electrophoresis, in accordance with the manufacturer's instructions of the kit as reported by Itoh et al. (2002).

Basal electrophoresis (no sample treatment / kit staining procedure), neuraminidase electrophoresis (neuraminidase treated sample / kit staining procedure), anti-intestinal ALP antibody electrophoresis (anti-intestinal ALP 
antibody treated sample / kit staining procedure), levamisole electrophoresis (no sample treatment / staining with substrate containing $4.2 \mathrm{~m} \mathrm{~mol} / 1$ levamisole hydrochloride) and WGA electrophoresis (supernatant after WGA precipitation as sample / kit staining procedure) were performed as described previously (Hatayama et al., 2011). In order to confirm the changes in serum ALP isoenzymes objectively, some gels were subjected to densitometry (QuickScan Densitometer, Helena Laboratories Corp., Saitama, Japan) and the activity of each band was calculated from the fraction ratio and serum ALP activity.

\section{Assay of ALP activity}

ALP activities of tissue extracts and serum were measured at $37^{\circ} \mathrm{C}$ using 4-nitrophenyl phosphate (4NPP) as substrate and 2-ethylaminoethanol (EAE) as buffer with an automatic analyzer TBA-120FR (Toshiba Medical Systems Corporation, Tokyo, Japan). In addition, in order to detect serum samples containing CALP, levamisole ((-)-Tetramisole hydrochloride, Sigma-Aldrich Co., St. Louis, MO, USA) was added to the ALP assay reagent at the final concentration of $4.2 \mathrm{mmol} / \mathrm{l}$ and the amount of inhibited ALP activity was measured.

\section{Statistical analysis}

Serum ALP activity obtained from un-fasted and fasted dogs is expressed as mean \pm S.D. Statistical analysis was performed using a paired Student's $t$-test (two-tailed) and correlation analysis was conducted using regression analysis in Microsoft Excel (Microsoft Corporation).

\section{RESULTS}

\section{Tissue ALP extracts}

Zymograms of tissue ALP extracts are shown in Fig. 1. In basal electrophoresis, liver, bone and small intestinal ALP bands were observed in that order from the anode (Fig. 1a). Neuraminidase electrophoresis caused a migration change of the liver and bone ALP bands to the cathode, but the small intestinal ALP band was unchanged (Fig. 1b). Anti-intestinal ALP antibody electrophoresis caused a disappearance of almost all the small intestinal ALP band in the resolving gel, and then two staining bands corresponding to the small intestinal ALP were observed at the both sides of stacking gel. However, the liver and bone ALP bands were unchanged (Fig. 1c). Levamisole electrophoresis caused almost all the staining of the liver and bone ALP bands to disappear, but the staining of the small intestinal ALP band remained (Fig. 1d).

\section{Serum liver and bone ALP isoenzymes}

Zymograms of serum ALP isoenzymes in intact dogs at 5 and 11 months old, and cholestasis model dogs are shown in Fig. 2. Basal electrophoresis showed band 1, band 2 and band 4 (atypical band) in all dogs, however, band 2 and 4 in 11-month-old dogs tended to decrease with age (Fig. 2a: gel 3 compared with gel 1), and the ALP activities of these isoenzymes were approximately $1 / 6$ those of 5-month-old dogs. In addition, band 4 shaped atypical form and individual difference was observed (a) $\mathrm{BE}$

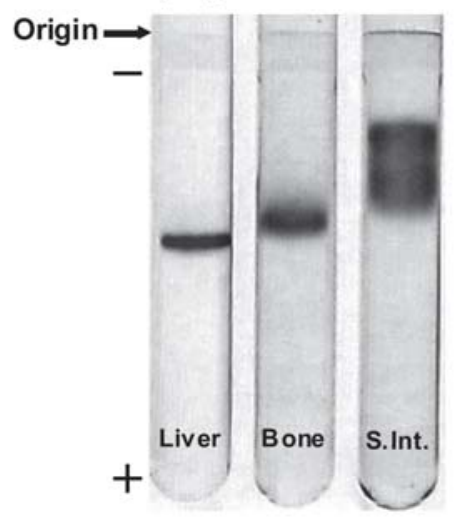

(b) $\mathrm{Ne}$

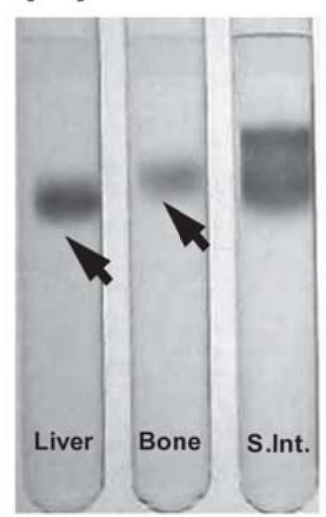

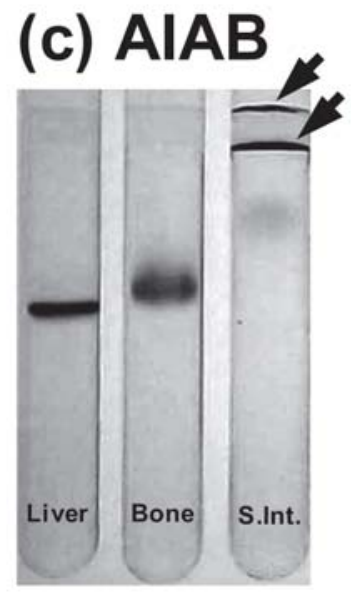
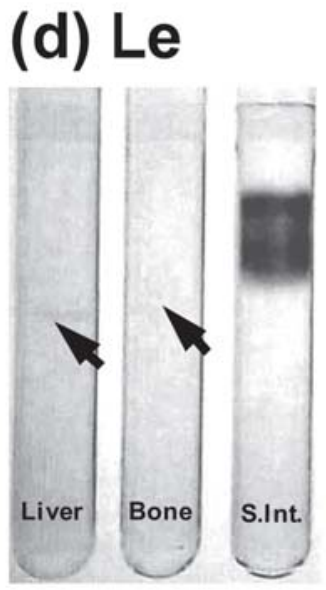

Fig. 1. Tissue ALP extracts. In order to examine the nature of main enzyme source of serum ALP, tissue ALP extracts from liver, bone and small intestine (S. int.) were electrophoresed by four methods as follows: (a) BE, basal electrophoresis; (b) Ne, neuraminidase electrophoresis; (c) AIAB, anti-intestinal ALP antibody electrophoresis; and (d) Le, levamisole electrophoresis. Oblique arrows indicate the significant changes. 
(a) Liver ALP and Bone ALP

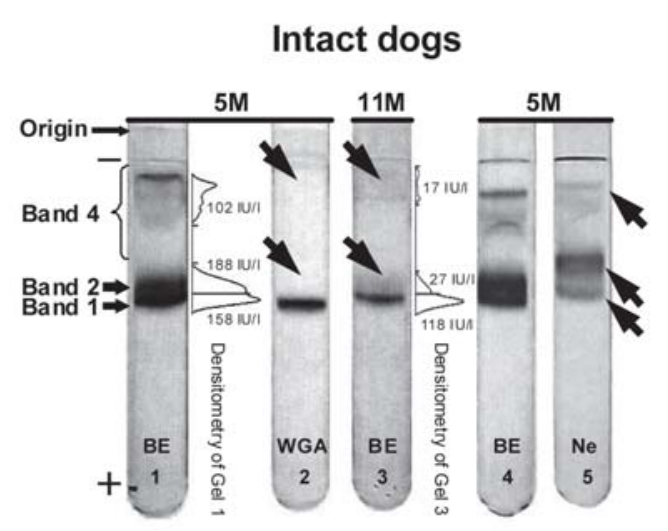

Cholestasis model ANIT $30 \mathrm{mg} / \mathrm{kg}$

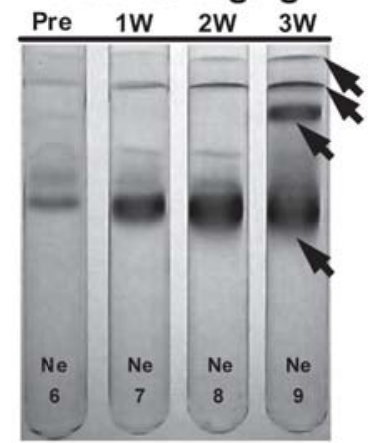

(b) WGA reactive ALP

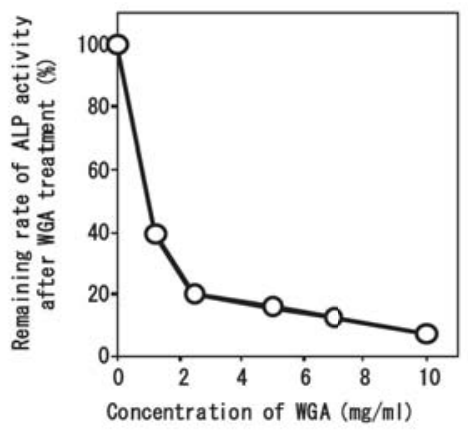

Fig. 2. Liver ALP and bone ALP in serum. (a) In order to detect liver ALP and bone ALP in serum, serum samples from 5 and 11-month-old intact beagle dogs, and a cholestasis model dogs (ANIT $30 \mathrm{mg} / \mathrm{kg}$ administration for 3 weeks) were electrophoresed by three methods as follows: BE, basal electrophoresis; WGA, WGA electrophoresis; and/or Ne, neuraminidase electrophoresis. Densitometry of the gels and the activity of each band calculated from the fraction ratio and serum ALP activity were shown on the right side of the gels. (b) In addition, remaining rate of ALP activity in the supernatant after $1-10 \mathrm{mg} / \mathrm{ml}$ WGA treated serum were examined. Oblique arrows indicate the significant changes.

(Fig. 2a: gel 1 and 4, samples were obtained individually). WGA electrophoresis caused disappearance of band 2 and band 4, and then only band 1 remained (Fig. 2a: gel 2). Neuraminidase electrophoresis caused migration change of all the bands to the cathode (Fig. 2a: gel 5 compared with gel 4). Band 2 largely migrated, however, band 1 migrated slightly, and then the separation between band 1 and band 2 became clear (Fig. 2a: gel 5). However, cholestasis model dogs showed a significant increase of band 1 with the increase in its degree in association with the elongation of the administration period (Fig. 2a: gels 7-9) and the appearance of a band in the resolving gel at the cathodal side (non-band 4) (Fig. 2a: gel 9). Moreover, staining was observed on both sides of stacking gel (Fig. 2a: gels 8 and 9, indicating they were high molecular ALP suggesting organ damage. In addition, the remaining rate of ALP activity in the supernatant after treatment of serum with WGA significantly decreased at the concentration of 1-5 mg/ml WGA (Fig. 2b). This result revealed the existence of WGA-reactive ALP isoenzyme in the dog serum.

Serum ALP isoenzymes which were observed as three bands in basal electrophoresis mentioned above were identified as follows: 1) Band 1 was liver ALP because this band shared homology with the tissue extract of liver ALP in relative migration and migration change, dominantly increased in cholestasis model dogs and was not reactive to WGA (Fig. 2a, Gel 2); 2) Band 2 was bone ALP because this band shared homology with the tissue extract of bone ALP in relative migration and migration change, was reactive to WGA (Fig. 2a, Gel 2), and tended to decrease with age (Fig. 2a: gel 3); and 3) Band 4, which showed atypical form with individual difference (Fig. 2a: gel 1 and 4) and had similar findings to band 2, was identified as bone-derived ALP (atypical ALP). Typical zymograms of liver ALP, bone ALP and atypical ALP are shown in Fig. 5.

\section{Serum intestinal ALP isoenzyme}

Zymograms of serum ALP isoenzymes in 5-month-old intact dogs are shown in Fig. 3. Levamisole electrophoresis caused almost all the staining in the resolving gels to disappear (Fig. 3a: gel 2 compared with gel 1). However, anti-intestinal ALP antibody electrophoresis caused no significant changes to the basal electrophoresis (Fig. 3a: gel 3 compared with gel 1). In addition, serum ALP activity in blood samples obtained before and after fasting from the same 6 males and 10 females was as follows: $151 \pm 73 \mathrm{IU} / 1$ before fasting and $148 \pm 79 \mathrm{IU} / 1$ after fasting. No significant changes of serum ALP activities were observed between before and after fasting $(p=0.2445)$. Therefore, intestinal ALP was not observed in the serum of laboratory beagle dogs.

\section{Serum corticosteroid-induced ALP isoenzyme (CALP)}

Zymograms of serum ALP isoenzymes in intact dogs 


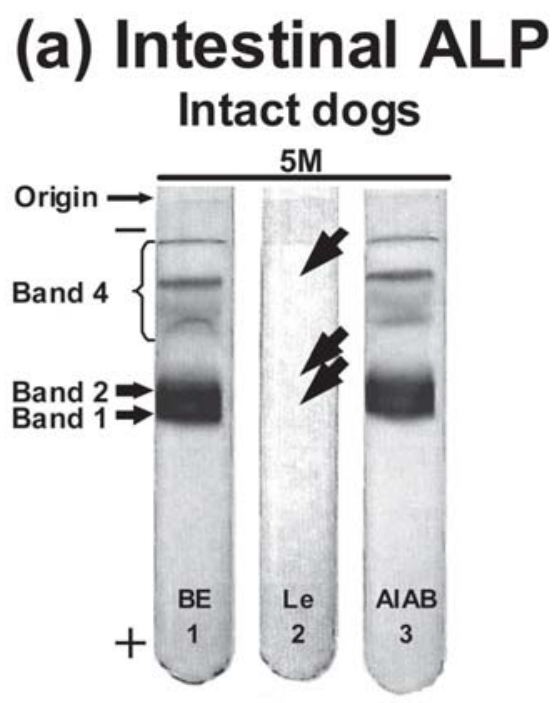

Fig. 3. Intestinal ALP in serum. (a) In order to detect intestinal ALP in serum, serum samples from 5-month-old intact beagle dogs were electrophoresed by three methods as follows: BE, basal electrophoresis; Le, levamisole electrophoresis; and AIAB, anti-intestinal ALP antibody electrophoresis. Oblique arrows indicate the significant changes.

which had an extra band (band 3) and those in cholestasis model dogs are shown in Fig. 4. Levamisole electrophoresis caused disappearance of almost all bands 1, 2 and 4 , and then only band 3 remained (Fig. 4a: gel 2 compared with gel 1). Neuraminidase electrophoresis caused a migration change of all bands to the cathode (Fig. 4a: gel 3 ), and band 3 still remained after neuraminidase plus levamisole electrophoresis (Fig. 4a: gel 4). Therefore, the band 3 was CALP because this band met the characteristics of CALP which were resistant to levamisole (intestinal-type ALP) and showed reduction in the anodal migration by neuraminidase (containing sialic acid). Typical zymogram of CALP is shown in Fig. 5.

In neuraminidase electrophoresis of serum samples from a cholestasis model, severe increases of band 1, which was broad to both sides, and band 3 were observed. Moreover, band 2, staining at the cathodal side of band 3 , staining at the most cathodal side of the resolving gel (non-band 4) and the anodal side of the stacking gel were also observed. Neuraminidase plus levamisole electrophoresis caused a significant decrease or disappearance of band 1 (oblique arrow (1)), band 2, staining at the cathodal side of band 3 (oblique arrow (2)) and staining at the anodal side of the stacking gel (oblique arrow (3)), however, band 3 (oblique arrow (4)) still remained (Fig. 4a: gel 6 compared to gel 5). Moreover, staining at the most cathodal side of the resolving gel also still remained (oblique arrow (5)).

In addition, in order to examine individual differences in the appearance of CALP, the correlation examination between serum ALP activity and the remaining activity after levamisole inhibition was performed. As the results show, there were some samples which located markedly deviated upwards from the regression line (appearance ratio: $7.1 \%$ for 5 to 10 months; $21.1 \%$ for 11 to 20 months; $26.7 \%$ for 21 to 30 months; $50.0 \%$ for 31 to 56 months) (Fig. 4b). They were examined by basal and levamisole electrophoresis. The result is that all samples showed band 3 by basal electrophoresis and band 3 still remained after levamisole electrophoresis as the zymograms of a representative sample showed (Fig. 4b). Based on these results, it became clear that there were individual differences in the appearance of band 3 and the appearance ratio became higher with age.

\section{DISCUSSION}

In this study, we demonstrated that the method we examined is useful for detecting serum ALP isoenzymes in laboratory beagle dogs.

Recently we reported that the "AlkPhor System" which is a commercially available PAG disk electrophoresis kit used in human ALP analysis is also suitable for detecting plasma ALP isoenzymes in mice, and this system is extremely useful to evaluate organ toxicity caused by certain drugs in toxicity studies (Hatayama et al., 2011). Although Itoh et al. (2002) also reported that this kit was useful for veterinary clinicopathological diagnosis in mongrel dogs, no simple and high resolution techniques of electrophoresis for analyzing ALP isoenzymes have been established for toxicity studies. In the present study, based on the report of Itoh et al. (2002), we tried to expand the application range of this kit to laboratory beagle dogs which are commonly used in toxicity studies.

In the present study, the main ALP isoenzyme in the serum of 5-month-old intact beagle dogs was bonederived which tended to decrease with age. On the other hand, the liver-derived isoenzyme greatly increased in cholestasis model dogs. This model dog also had the band which was high molecular weight and/or resistant to levamisole treatment. Individual differences were observed in the appearance of CALP.

In tissue ALP extracts, liver, bone and small intestinal ALP bands were observed in that order from the anode in agreement with the findings previously reported in mongrel dogs (Itoh et al., 2002), but the bands of liver and bone previously reported were broad. This suggests that 


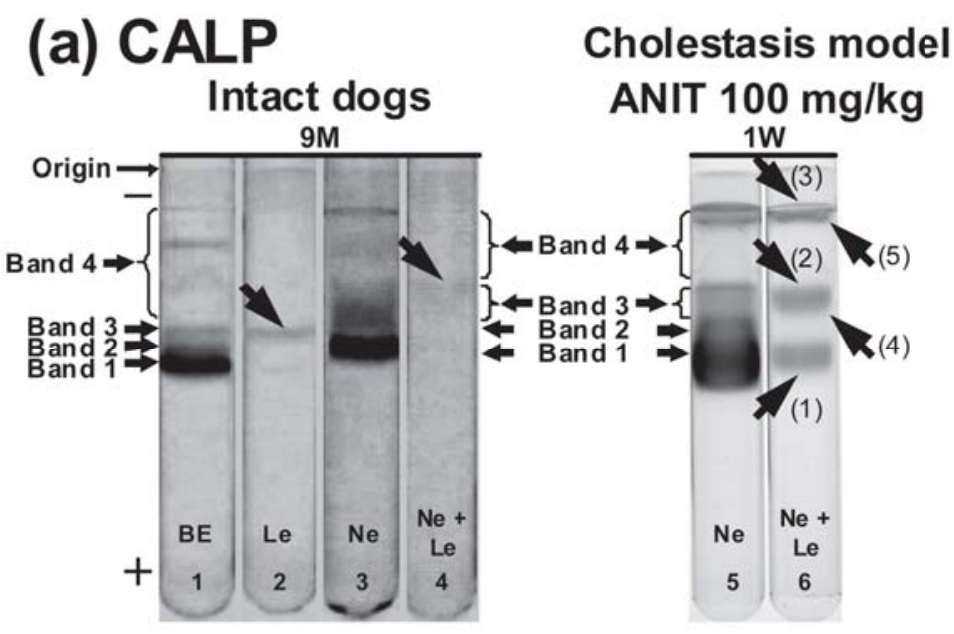

\section{(b) Individual difference of CALP}

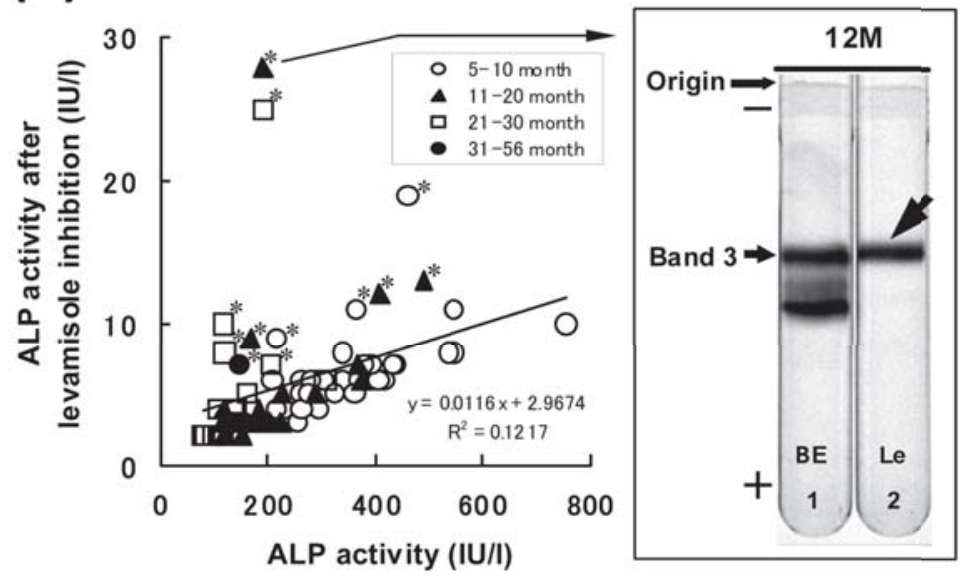

Fig. 4. Corticosteroid-induced ALP (CALP) in serum. (a) In order to examine CALP in serum, a serum sample which showed extra band in 9-month-old intact beagle dogs and that of a cholestasis model dog (ANIT $100 \mathrm{mg} / \mathrm{kg}$ administration for 1 week) were electrophoresed by four methods as follows: BE, basal electrophoresis; Le, levamisole electrophoresis; Ne, neuraminidase electrophoresis; and/or $\mathrm{Ne}+\mathrm{Le}$, the combination of neuraminidase and levamisole electrophoresis (neuraminidase treated sample / staining with substrate containing $4.2 \mathrm{mmol} / 1$ levamisole hydrochloride). (b) In addition, in order to examine individual differences of the occurrence of CALP, the correlation between serum ALP activity and the remaining activity after levamisole inhibition were examined. Samples which markedly deviated upwards from the regression line $(5$ males and 7 females, marked with *) were electrophoresed by basal electrophoresis and levamisole electrophoresis, and the zymograms of the representative sample were shown. Oblique arrows indicate the significant changes.

our method of extraction was effective to sharpen these bands. On the other hand, although the band of small intestinal ALP in the previous report was single (Itoh et al., 2002), one more band was observed at the anodal side in the present study. In addition, two staining bands corresponding to small intestinal ALP were observed at both sides of the stacking gel after anti-intestinal ALP antibody treatment. Since we collected tissue samples from duodenum and jejunum to obtain small intestinal ALP extract in the present study, there might be two intestinal ALP isoenzymes in dogs as is the case in rats (Engle and Alpers, 1992; Xie and Alpers, 2000). As a result, the zymogram of each tissue ALP extract was clear without tailing. The small intestinal ALP extract was irresponsive to neuraminidase, responsive to anti-intestinal ALP antibody and resistant to levamisole opposite to the liv- 
Dog serum ALP isoenzymes in toxicity studies by PAG

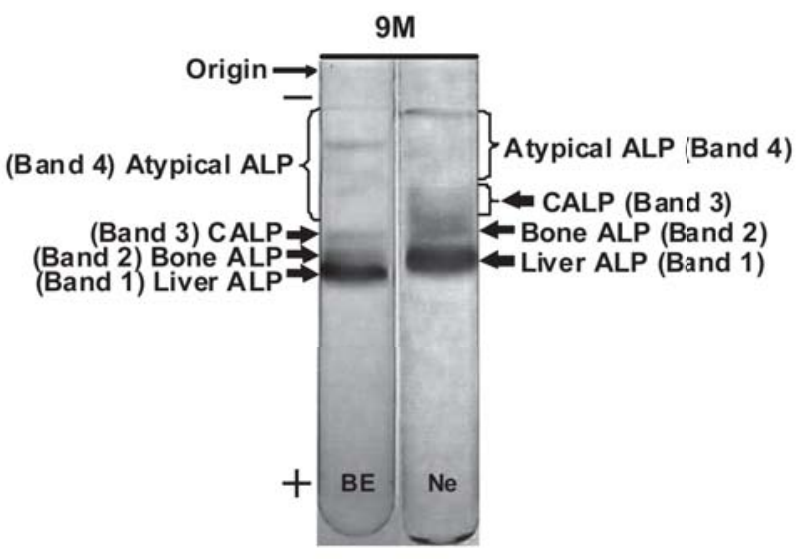

Fig. 5. Typical zymograms of serum ALP on 9-month-old intact laboratory beagle dogs with corticosteroid-induced ALP by basal electrophoresis (BE) and neuraminidase electrophoresis $(\mathrm{Ne})$ were shown.

er and bone ALP extracts and these findings were newly confirmed in this study. The nature of tissue ALP extracts was easily used as markers for the identification of plasma ALP isoenzymes.

Although the electrophoretic mobility of serum isoenzymes were not constant in mongrel dogs (Itoh et al., 2002), those of laboratory beagle dogs were constant. Therefore, we could show typical zymograms of serum ALP (Fig. 5) and that was newly confirmed in this study. In dog serum, the existence of WGA-reactive ALP isoenzymes was confirmed, and bone ALP disappeared in WGA electrophoresis. Human bone ALP has been reported to be precipitated with WGA (Rosalki and Foo, 1984; Behr and Barnet, 1986), and we reported that mouse bone ALP also showed WGA-reactivity (Hatayama et al., 2011). Since the separation between serum liver ALP and bone ALP became clear by neuraminidase electrophoresis in agreement with findings previously reported in humans (Moss and Edwards, 1984) and mice (Hatayama et al., 2011), pre-treatment with neuraminidase was thought to be necessary for sufficient resolution of liver ALP isoenzymes. Although the activities tended to decrease with age in both sexes, the main portion of serum ALP activity was the bone-derived isoenzyme in intact 5-month-old beagle dogs (data not shown). Since atypical ALP showed the same characteristics as that of the bone-derived one, it was identified as bone-type ALP corresponding to human variant bone ALP isoenzyme. Similar findings were also reported in humans and mongrel dogs (Van Hoof et al., 1990; Itoh et al., 2002).

On the other hand, serum liver ALP was approximately a quarter of total serum ALP in 5-month-old intact dogs and it tended to increase with age (data not shown) as previously reported in dogs (Syakalima et al., 1998). Serum liver ALP isoenzyme remarkably increased in cholestasis model dogs and that was newly cofirmed in this study as previously reported in mice (Hatayama et al., 2011).

Since it is well known in humans that an increase in small intestinal ALP is associated with ingestion of high fat diets (Matsushita et al., 2002), serum ALP activity was compared between un-fasted and fasted dogs. Serum ALP activity was found to be similar between un-fasted and fasted dogs. Namely, no reaction to anti-small intestinal ALP antibody was noticed and no band remained after levamizole inhibition in the serum of intact dogs. These findings indicated that almost no small intestinal ALP was detected in the serum of intact dogs. It has been reported that intestinal ALP has never been detected due to its very short half-life of 3-6 minutes (Syakalima et al., 1998).

It was reported that CALP was intestinal-type ALP produced in the liver, which was resistant to levamisole, and it had sugar chains containing sialic acid, which showed a reduction in the anodal migration by neuraminidase on electrophoresis (Wellman et al., 1982; Hoffmann et al., 1988; Syakalima et al., 1998). As a result, since a band at the cathodal side of bone ALP met the characteristics of CALP as mentioned above, it was identified as CALP in the present study. Moreover, it became clear that the appearance of CALP showed individual differences and that was newly confirmed in this study. The appearance ratio became higher with age as previously reported (Itoh et al., 2002).

However, in the cholestasis model dogs, various types of high molecular ALPs were observed. No cleavage of GPI anchor-containing ALP and/or their aggregate, and complex with other lipid-rich molecules would appear in serum especially in hepatobiliary disease (Moss, 1992). Especially, in the animals treated with ANIT $100 \mathrm{mg} / \mathrm{kg}$, staining at the cathodal side of CALP and staining at the anodal side of the stacking gel inhibited by levamisole were tissue non-specific ALP which might be presumptive of liver-derived isoenzymes. On the other hand, staining at the most cathodal side of the resolving gel (Fig. 4, oblique arrow (5)) which was resistant to levamisole (non-atypical ALP) might indicate the appearance of polymerized small intestinal ALP and/or CALP. In the present study, bone and liver ALP extracts were strongly inhibited by levamisole, while the small intestinal ALP extract resisted as previously reported in humans (Van Belle, 1976) and mice (Hatayama et al., 2011). Although small intestinal ALP is not detected in intact dogs and CALP is not always detected, the concomitant use of 
levamisole in routine laboratory tests could be valuable in revealing possible increases in these small intestinal-type ALPs in pathological conditions such as cholestasis and these findings were newly confirmed in this study.

In laboratory beagle dogs, it was reported that there were no differences in serum ALP activity between sexes and among breeding colonies (Watanabe et al., 2008). There also was no sex difference observed in any ALP isoenzyme in the present study on beagle dogs (data not shown), while a sex difference was detected in bone ALP isoenzyme in ICR mice (Hatayama et al., 2011).

Since it was not possible to differentiate the high molecular ALP which was resistant to levamisole from intestinal ALP or CALP, further investigation is required. Moreover, further accumulation of information of serum ALP isoenzymes under various pathogenic conditions is expected.

In conclusion, the commercially available PAG disk electrophoresis kit used in human ALP analysis (AlkPhor System) is suitable for detecting serum ALP isoenzymes in laboratory beagle dogs, and this method is considered to be extremely useful to evaluate organ toxicity caused by certain drugs in toxicity studies.

\section{ACKNOWLEDGMENTS}

The authors would like to thank Dr. Kunio Doi, Emeritus Professor of the University of Tokyo, for reviewing this paper and Mr. Pete Aughton, D.A.B.T., Dip.R.C.Path., ITR Laboratories Canada Inc., for proofreading.

\section{REFERENCES}

Behr, W. and Barnert, J. (1986): Quantification of bone alkaline phosphatase in serum by precipitation with wheat-germ lectin: a simplified method and its clinical plausibility. Clin. Chem., 32, 1960-1966.

Dingjan, P.G., Postma, T. and Stroes, J.A.P. (1973): Quantitative differentiation of human serum alkaline phosphatase isoenzymes with polyacrylamide disc gel electrophoresis. Z. Klin. Chem. Klin. Biochem., 11, 167-171.

Engle, M.J. and Alpers, D.H. (1992): The two mRNAs encoding rat intestinal alkaline phosphatase represent two distinct nucleotide sequences. Clin. Chem., 38, 2506-2509.

Hatayama, K., Nishihara, Y., Kimura, S., Goto, K., Nakamura, D., Wakita, A. and Urasoko, Y. (2011): Alkaline phosphatase isoenzymes in mouse plasma detected by polyacrylamide-gel disk electrophoresis. J. Toxicol. Sci., 36, 211-219.

Hoffmann, W.E., Sanecki, R.K. and Dorner, J.L. (1988): A technique for automated quantification of canine glucocorticoidinduced isoenzyme of alkaline phosphatase. Vet. Clin. Pathol., 17, 66-70.

Hoshiya, T., Watanabe, D., Ishii, T., Yamaguchi, Y., Matsuoka, T., Saito, H., Hatayama, K., Nagashima, Y., Okaniwa, A. and
Yoshikawa, T. (2002): Comparison of pathologic features in experimental hyperphosphatasemic conditions in beagle dogs treated with $\alpha$-naphthylisothiocyanate and phenobarbital. J. Toxicol. Pathol., 15, 49-59.

Itoh, H., Kakuta, T., Genda, G., Sakonju, I. and Takase, K. (2002): Canine serum alkaline phosphatase isoenzymes detected by polyacrylamide gel disk electrophoresis. J. Vet. Med. Sci., 64, 35-39.

Matsushita, M., Irino, T., Kawaguchi, T. and Komoda, T. (2002): The effect of different buffers and amounts of intestinal alkaline phosphatase isoforms on total alkaline phosphatase activity. Clin. Chim. Acta, 319, 49-55.

Moss, D.W. and Edwards, R.K. (1984): Improved electrophoretic resolution of bone and liver alkaline phosphatases resulting from partial digestion with neuraminidase. Clin. Chim. Acta, 143, 177-182.

Moss, D.W. (1992): Perspectives in alkaline phosphatase research. Clin. Chem., 38, 2486-2492.

Rosalki, S.B. and Foo, A.Y. (1984): Two new methods for separating and quantifying bone and liver alkaline phosphatase isoenzymes in plasma. Clin. Chem., 30, 1182-1186.

Syakalima, M., Takiguchi, M., Yasuda, J. and Hashimoto, A. (1998): The canine alkaline phosphatases: A review of the isoenzymes in serum, analytical methods and their diagnostic application. Jpn. J. Vet. Res., 46, 3-11.

Van Belle, H. (1976): Alkaline phosphatase. I. Kinetics and inhibition by levamisole of purified isoenzymes from humans. Clin. Chem., 22, 972-976.

Van Hoof, V.O., Hoylaerts, M.F., Geryl, H., Van Mullem, M., Lepoutre, L.G. and De Broe, M.E. (1990): Age and sex distribution of alkaline phosphatase isoenzymes by agarose electrophoresis. Clin. Chem., 36, 875-878.

Watanabe, D., Hori, H., Ishii, T., Ishigami, M., Mizuguchi, H., Ishikawa, T., Hoshiya, T., Okaniwa, A. and Oyamada, T. (2008): Age-dependent changes in the activity of serum alkaline phosphatase in laboratory beagle dogs. J. Toxicol. Sci., 33, 241-244.

Wellman, M.L., Hoffmann, W.E., Dorner, J.L. and Mock, R.E. (1982): Comparison of the steroid-induced, intestinal, and hepatic isoenzymes of alkaline phosphatase in the dog. Am. J. Vet. Res., 43, 1204-1207.

Xie, Q. and Alpers, D.H. (2000): The two isozymes of rat intestinal alkaline phosphatase are products of two distinct genes. Physiol. Genomics, 3, 1-8. 